\title{
Benign periarticular, bone and joint lipomatous lesions
}

Si Wei Kheok ${ }^{1}$, MBBS, FRCR, Keh Oon $\underline{O n g}^{2}$, MRCS, FRCR

\begin{abstract}
Benign periarticular, bone and joint lipomatous lesions are rare entities that are increasingly being identified using current imaging techniques. This pictorial review illustrates the wide range of imaging presentations of these lesions at various sites and their pathognomonic features. The main lesions reviewed include intraosseous lipoma, liposclerosing myxofibrous tumour, lipoma arborescens and intra-articular lipoma.
\end{abstract}

Keywords: benign neoplasms, bone neoplasms, lipoma, soft tissue neoplasms

\section{INTRODUCTION}

Benign periarticular, bone and joint lipomatous lesions affect people of a wide range of ages - from the late paediatric to the geriatric populations, with an average age of 40-60 years. ${ }^{(1-5)}$ These lesions are rare and tend to have either an equal maleto-female distribution or a slight male preponderance. ${ }^{(1,3)}$ With the advent of advanced imaging modalities, benign lesions are increasingly being characterised. Thus, it is important to recognise their imaging features, so as to avoid unnecessary biopsies. In this review, we outline the clinical presentations of benign periarticular, bone and joint lipomatous lesions and illustrate their distinctive imaging features.

\section{INTRAOSSEOUS LIPOMA}

Intraosseous lipoma is a rare, benign bone lesion with a cited prevalence of less than $0.1 \%$ of bone tumours reported. ${ }^{(1,6)}$ The true prevalence in the general population may be higher, as this lesion is commonly asymptomatic and its radiographic features may lead to it being labelled as benign without further characterisation. Pathologic interpretation of this lesion in the absence of radiological correlation may sometimes be misleading, as histologically, an intraosseous lipoma can be difficult to differentiate from normal fat in yellow marrow or from osteonecrosis. ${ }^{(1)}$ There is a slight male preponderance, with one meta-analysis reporting a male-to-female ratio of $4: 3$. $^{(2)}$ There is a wide age range at first presentation (5-85 years), with the majority affected in their fourth to fifth decades of life. ${ }^{(1,2)}$

Clinically, approximately half of the patients with intraosseous lipoma are asymptomatic. ${ }^{(7)}$ Symptomatic patients may present with pain, which has been hypothesised to be due to expansile remodelling of the bone or the related ischaemic changes that accompany such lesions; alternatively, the pain may be purely incidental, such as when the pain trigger arises from a nearby pathology ${ }^{(1)}$ Swelling and pathological fractures are rare clinical presentations. $^{(1,2)}$

Commonly affected sites include: the calcaneum (8\%) (Figs. 1-3); intertrochanteric or subtrochanteric regions of the femur (34\%) (Fig. 4); proximal tibial metaphysis (13\%) (Fig. 5); pelvis (8\%) (Fig. 6); and the spine (Fig. 7). Other reported sites
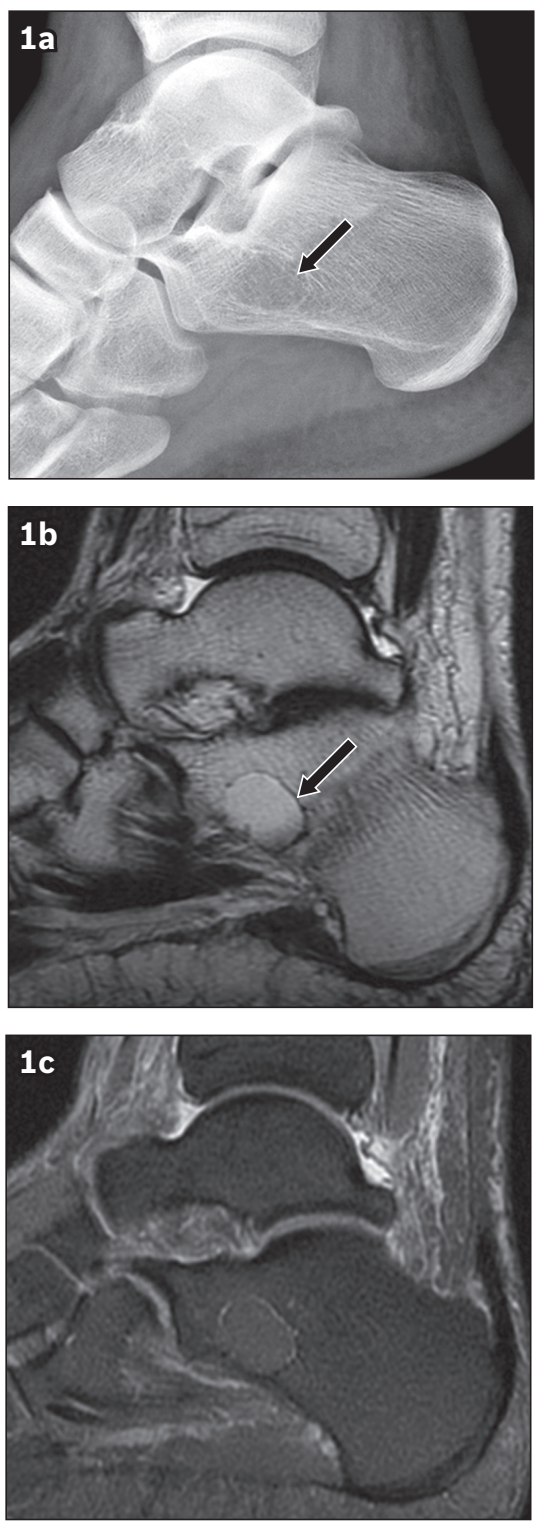

Fig. 1 Stage 1 intraosseous lipoma of the calcaneum. (a) Lateral radiograph of the ankle shows a well-defined lucent lesion in the calcaneum. The lesion has a sclerotic rim that may be due to capsular or peripheral calcification (arrow). (b) Sagittal T2-W and (c) T2-W fat-saturated MR images show that the lesion is isointense to subcutaneous fat, with complete fat suppression. No cystic change or calcification is detected. The hypointense rim (arrow in b) corresponds to the sclerotic rim seen on the radiograph. 

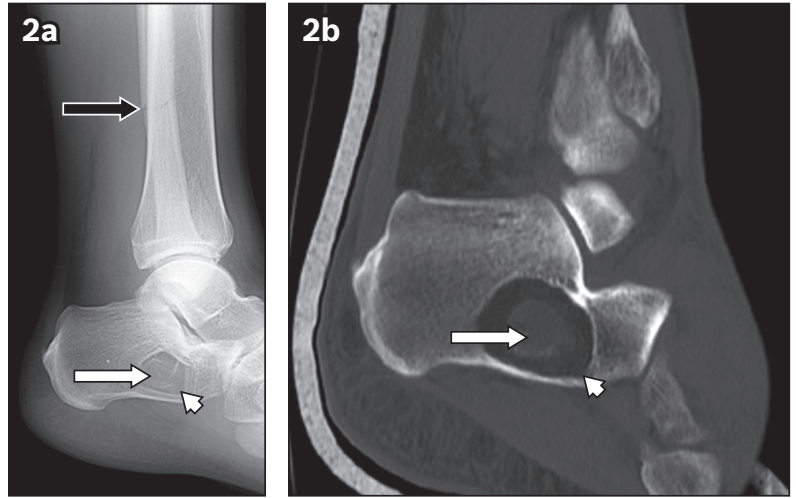

Fig. 2 Stage 2 intraosseous lipoma of the calcaneum. (a) Lateral radiograph shows a distal fibula fracture (black arrow) and an incidental, well-defined, radiolucent lesion at the critical angle of the calcaneum (arrowhead). Both the radiograph and (b) sagittal CT image of the ankle show that the lesion has similar density (arrowhead) as subcutaneous fat and contains internal calcifications (white arrows).

include: the proximal and distal fibular metaphyses; humerus (Fig. 8); skull; mandible; rib; and radius. ${ }^{(1,2)}$ Intraosseous lipoma can extend from the metaphysis to the diaphysis or epiphysis, although the epiphysis is an uncommon site. ${ }^{(1,2)}$

A key imaging feature of intraosseous lipoma is the presence of fat, which has radiological characteristics that are similar to subcutaneous fat on all modalities. Hence, radiographically, the lesion typically appears radiolucent (Fig. 1).(1,2,6) On computed tomography (CT), it has a fat density of $-60 \mathrm{HU}$ to $-100 \mathrm{HU}^{(1)}$

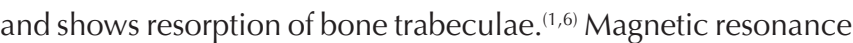
(MR) imaging shows the lesion to be isointense to subcutaneous fat on T1- and T2-weighted images, and hypointense on fat-saturated sequences. Compared to the surrounding normal bone marrow, the lesion has mildly higher T1-weighted signal intensity. In addition, a thin hypointense rim due to capsular or peripheral ossification may be present. ${ }^{(1,6)}$ Although rare, expansile remodelling of the bone (Fig. 6), which has been described in the fibula, spine and sacrum, is also a useful feature. ${ }^{(2)}$

Milgram describes three types of intraosseous lipoma: Stage 1 lesions consist of only viable fat cells (Fig. 1); Stage 2 lesions contain viable fat cells as well as fat necrosis and calcification (Fig. 2); and Stage 3 lesions have necrotic fat, calcification of necrotic fat, cysts and reactive woven bone (Fig. 3). ${ }^{(7)}$ The radiological findings of the various stages reflect that of their histological components. The imaging features of the lipomatous component of these lesions have been described earlier. Fat necrosis, which is seen in Stage 2 and 3 lesions, is hyperdense on $\mathrm{CT} .{ }^{(7)} \mathrm{On} \mathrm{MR}$ imaging, fat necrosis has variable T1-weighted signal and is hyperintense on T2-weighted images ${ }^{(6)}$ Cystic formations are hypointense on T1-weighted images and hyperintense on T2-weighted images. Calcifications within the central portion of Stage 2 and 3 intraosseous lipomas appear as signal voids on MR imaging. Haemorrhage, which is exceedingly rare, is hyperintense on T1-weighted images with or without fatsaturation and hypointense on T2-weighted images.(8) On bone scintigraphy, intraosseous lipomas demonstrate absent-to-moderate uptake of radionuclide (Fig. 6b). ${ }^{(9)}$

Intraosseous lipoma of the calcaneum has a classical appearance. It usually occurs at the critical angle of the
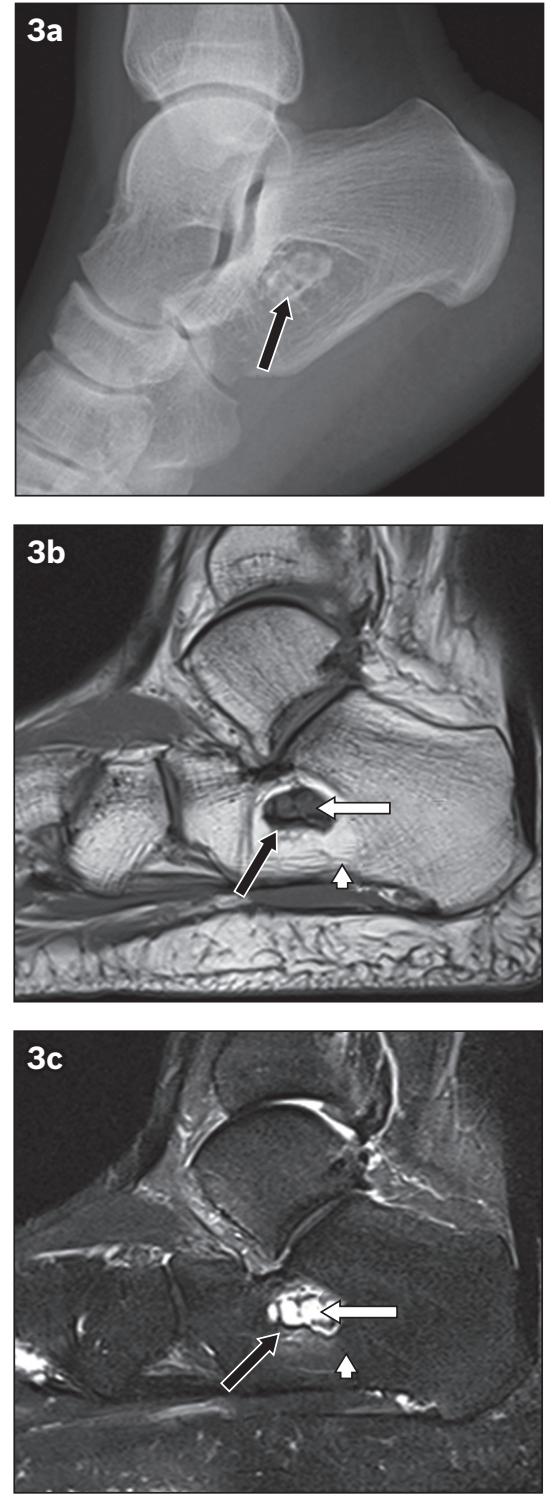

Fig. 3 Stage 3 intraosseous lipoma of the calcaneum. (a) Lateral radiograph shows the classical appearance at the critical angle of the calcaneum with internal calcifications (black arrow). (b) Sagittal T1-W and (c) short tau inversion recovery MR images show lipomatous content (arrowheads), persistent hypointensity of the internal calcifications (black arrows in b \& c) and cystic changes (white arrows).

calcaneum, which is an area of increased porosity that is physiological. Compared to intraosseous lipomas at other sites, calcaneal lipomas show a greater prevalence of marginal sclerosis $(61 \%)$ and internal calcification $(62 \%)$, and rarely cause bone expansion. ${ }^{(2)}$ Calcification within a lucent lesion at the critical angle of the calcaneum, which is pathognomonic of intraosseous lipoma, helps to differentiate it from unicameral bone cysts. ${ }^{(1)}$

Differentiating intraosseous lipoma from osteonecrosis on CT and MR imaging may be difficult at times, as they both contain fat and are separated from the surrounding marrow by a rim of tissue. ${ }^{(1)}$ Distinguishing features of intraosseous lipoma include resorption of bone trabeculae, a rounded (rather than serpentine) margin and expansile remodelling. ${ }^{(1,6)}$ Radiographically, intraosseous lipoma may be mistaken for fibrous dysplasia. However, fibrous dysplasia is hypointense on T1-weighted images and demonstrates heterogeneous enhancement, and its epicentre is often at the 

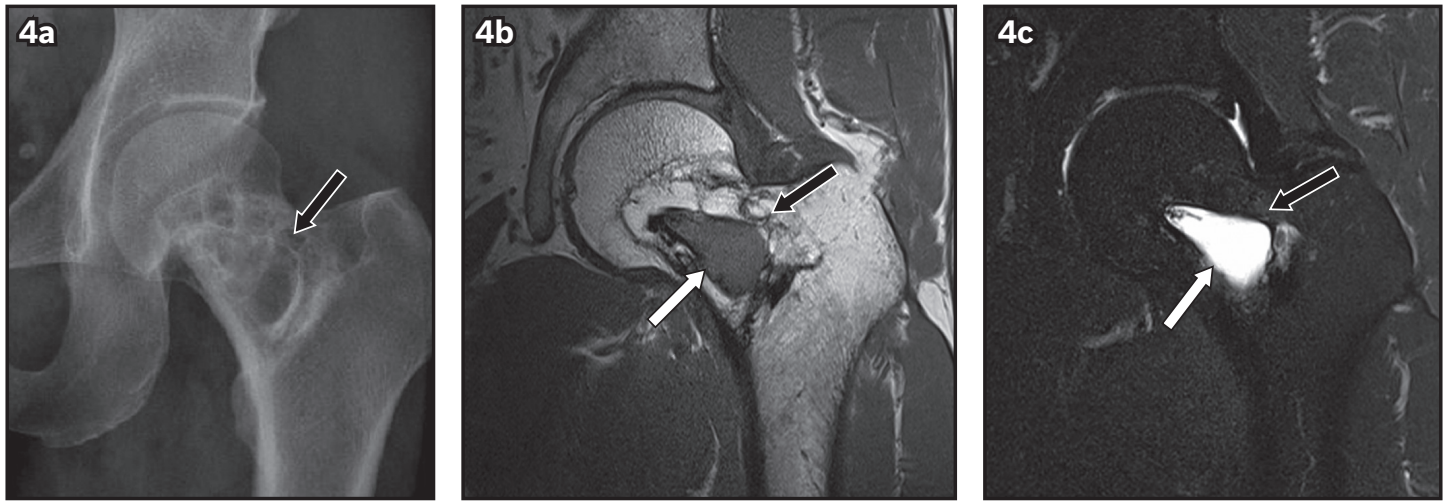

Fig. 4 Stage 3 intraosseous lipoma of the femur. (a) Anteroposterior radiograph shows a multiseptated lucent lesion in the left femoral neck (black arrow), which may be mistaken for fibrous dysplasia or an aneurysmal bone cyst. (b) T1-W and (c) T2-W fat-saturated MR images show the lipomatous lesion (black arrows in b \& c) in the femoral neck with a cystic component (white arrows).
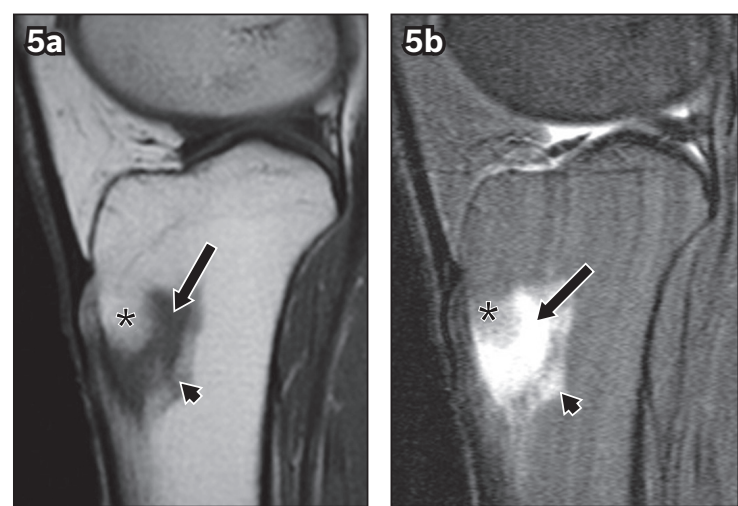

Fig. 5 Intraosseous lipoma of the tibia. (a) Sagittal T1-W and (b) T2-W fat-saturated MR images show that the proximal component of the lesion $\left(^{*}\right)$ is isointense to subcutaneous fat. The inferior component is hypointense on the T1-W image and hyperintense on the T2-W fat-saturated image, which is consistent with cystic degeneration (arrows). Mild perilesional oedema is also demonstrated (arrowheads).

diaphysis. Other differential diagnoses include osteoblastoma, enchondroma, chondroblastoma, chondrosarcoma, Brodie abscess, non-ossifying fibroma and giant cell tumour.(7)

Intraosseous lipomas are usually solitary lesions, although multiple lesions have been described.(2) It is important to distinguish them from multiple lipomatosis, which, unlike intraosseous lipoma, is not encapsulated by connective tissue and may be due to endocrinological abnormalities such as Type IV hyperlipoproteinaemia. ${ }^{(10)}$ A conservative approach to the management of asymptomatic or incidentally discovered intraosseous lipomas is often taken. Symptomatic lesions have been treated successfully with curettage and bone graft placement. ${ }^{(7)}$ They rarely recur after treatment and malignant transformation is uncommon. ${ }^{(1)}$

\section{LIPOSCLEROSING MYXOFIBROUS TUMOUR}

Liposclerosing myxofibrous tumour (LSMFT) is a benign fibroosseous lesion that consists of a wide range of histological components, including myxoid change, lipoma, fat necrosis, fibroxanthoma, fibrosis, reactive bone, ischaemic ossification, calcification and fibrous dysplasia-like features. ${ }^{(1,3,4)}$ The origin of LSMFT is contentious. Some authors regard LSMFT as a manifestation of an underlying well-known bone entity with varying degrees of degeneration. Histological and genetic studies suggest that the underlying bone entity may be fibrous dysplasia or intraosseous lipoma. ${ }^{(4)}$ Others, however, consider it a distinct entity. ${ }^{(3)}$

LSMFT affects an equal proportion of men and women. The age of presentation ranges from 15 to 80 years. ${ }^{(3,4)}$ In Kransdorf et al's study, $85 \%$ of the lesions occurred in the femur, with $91 \%$ of these being located at the intertrochanteric region (Fig. 9). Other uncommon sites include the femoral shaft, ilium, humerus and rib. ${ }^{(3)}$ LSMFT is often asymptomatic or may present with pain. Occasionally, patients may present with pathological fractures. ${ }^{(3)}$ Radiographically, LSMFT has a geographic lucent appearance with a sclerotic margin, in the presence or absence of expansile remodelling. ${ }^{(1,3,4)}$ Mineralised matrix is often present. On bone scintigraphy, it has increased radionuclide uptake, but to a lesser degree than in fibrous dysplasia. ${ }^{(1,3)}$ Interestingly, despite the presence of lipomatous tissue (however small the amount), the lesion does not have lipomatous characteristics on CT or MR imaging, likely because the lipomatous tissue has intermixed with myxofibrous or fibro-osseous tissue. ${ }^{(1)} \mathrm{OnCT}$, its non-mineralised regions have a higher density than fat, but a lower density than skeletal muscle. On MR imaging, it is moderately homogeneous, and appears isointense to skeletal muscle on T1-weighted images and hyperintense on T2-weighted images. ${ }^{(3)}$ Differential diagnoses of LSMFT include intraosseous lipoma and fibrous dysplasia. Malignant transformation has been described in 10\%-16\% of cases, the majority of which are osteosarcomas. ${ }^{(1,3)}$ Other malignancies that LSMFT can transform into include malignant fibrous histiocytoma, ${ }^{(1,3)}$ malignant fibroxanthomas and high-grade spindle cell tumours.

Treatment of LSMFT depends on its presentation. Asymptomatic lesions that are discovered incidentally may not warrant treatment, while symptomatic lesions may be treated with curettage, bone grafting and fixation. ${ }^{(1)} \mathrm{A}$ high level of suspicion for malignancy should be maintained if the lesion has aggressive features or recurs quickly after surgical resection.

\section{LIPOMA ARBORESCENS}

Lipoma arborescens is a rare intra- or extra-articular ${ }^{(5,11)}$ benign lesion with villous synovial proliferation and subsynovial 

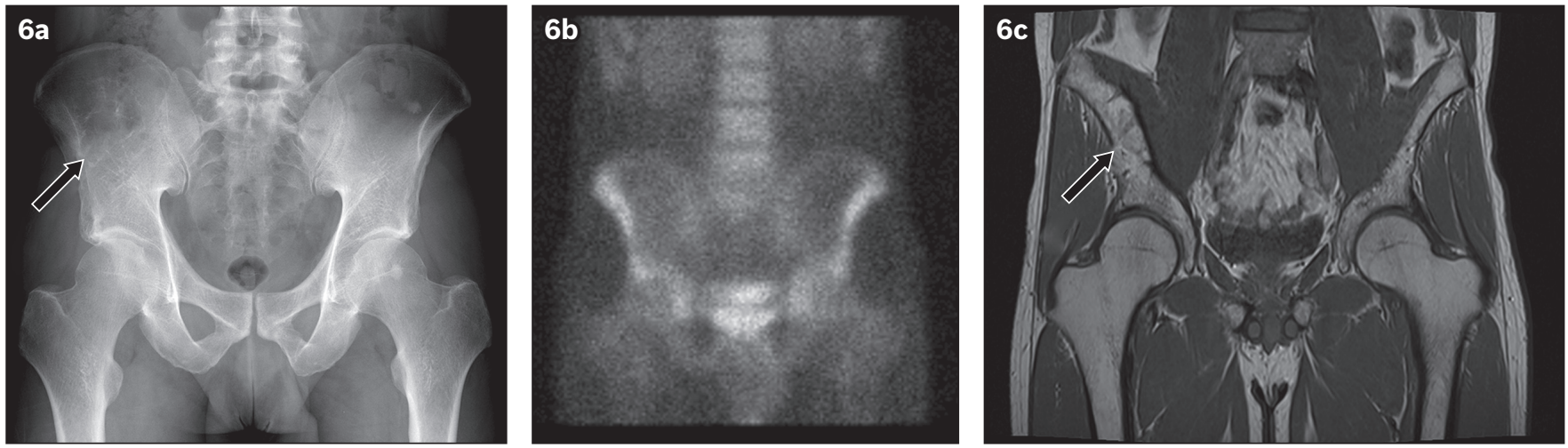

Fig. 6 Intraosseous lipoma of the pelvis. (a) Anteroposterior radiograph shows a multiseptated lucent lesion in the right ilium (arrow). (b) Technetium$99 \mathrm{~m}$ scintigraphy image shows no increase in radiotracer uptake. (c) Coronal T1-W MR image shows an expansile lesion with lipomatous content (arrow).
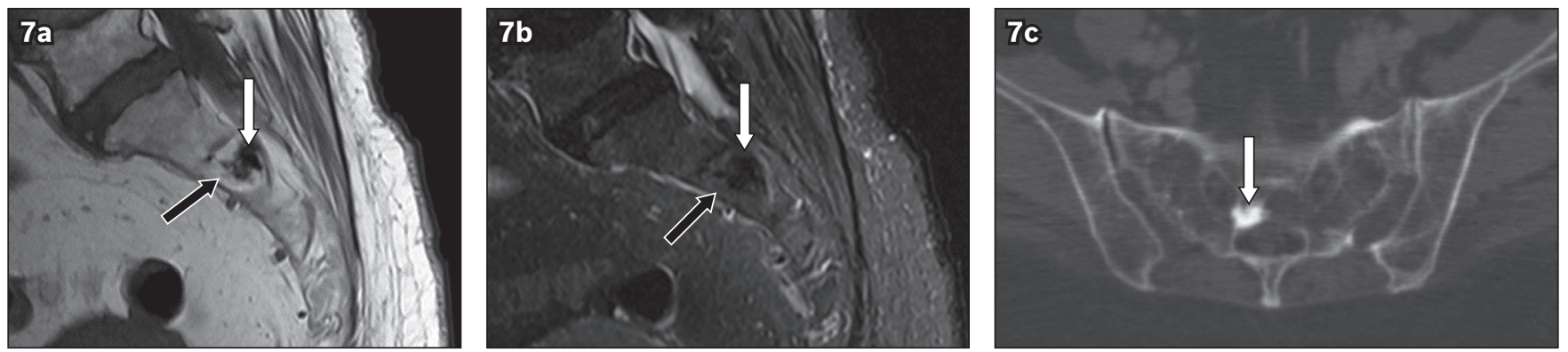

Fig. 7 Intraosseous lipoma of the sacrum. (a) Sagittal T1-W and (b) short tau inversion recovery (STIR) MR images show a T1-hyperintense lesion, with signal suppression on the STIR image (black arrows) in the second sacral vertebra, consistent with a lipoma. Its hypointense centre is due to central calcification (white arrows in a \& b), which can also be identified on both radiograph (not shown) and (c) axial CT image of the pelvis (white arrow).
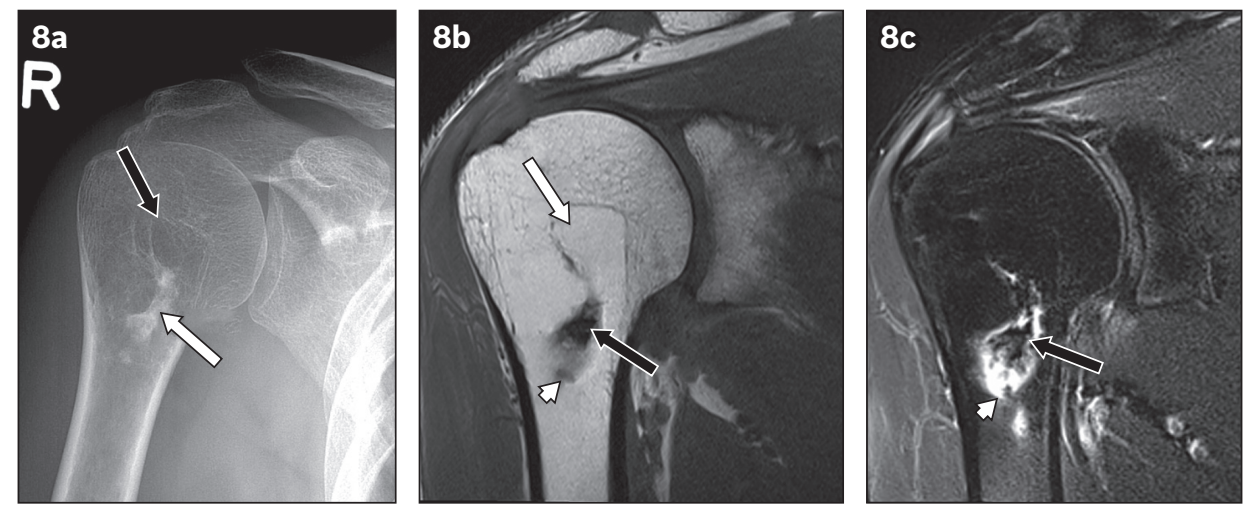

Fig. 8 Stage 3 intraosseous lipoma in the right humeral neck. (a) Radiograph shows a lucent lesion (white arrow) with central calcification (black arrow). (b) T1-W MR image shows lipomatous tissue (white arrow) that is differentiated from adjacent bone marrow by the absence of trabeculae and higher signal intensity. Central calcifications are hypointense on both T1-W and (c) T2-W fat-suppression sequence (black arrows in b \& c). Cystic degeneration (arrowheads) of the lesion is in keeping with Stage 3 intraosseous lipoma.

replacement of fat. It affects patients across a wide age range (15-90 years) $)^{(5)}$ with an average age of 50 years..$^{(5,11)}$ The gender distribution of affected patients is almost equal. ${ }^{(5)}$ Patients may present clinically with stiffness, swelling or pain. ${ }^{(11)}$

In a study by Howe and Wenger, ${ }^{(5)} 72 \%$ of cases of lipoma arborescens were cases of monoarticular involvement of the knee (Fig. 10). Other sites of involvement included the shoulder (Fig. 11); the elbow or bicipitoradial bursa (Fig. 12); extensor compartment of the wrist; the hip; and the ankle. ${ }^{(5)}$ Polyarticular involvement is rare. These lesions are often associated with degenerative or inflammatory arthritis. ${ }^{(5,11)}$ Lesions that involve the shoulder are associated with rotator cuff tears. ${ }^{(5)}$

Most cases of lipoma arborescens are diffuse, although onefifth of them present as pseudomasses. ${ }^{(11)}$ Radiographically, soft tissue swelling in the joint may be detected. ${ }^{(12)} \mathrm{On} \mathrm{CT}$, the soft tissue lesion appears as fat attenuation. Its villous appearance on CT may not be as well appreciated as it would be on MR imaging. On MR imaging, the key imaging feature is the presence of a frond-like synovial lesion with high signal intensity on T1- and T2-weighted images, similar to subcutaneous fat, ${ }^{(12)}$ which is suppressed on fat-saturated or short tau inversion recovery sequences (Figs. 10-12). ${ }^{(11-13)}$ The lesion does not enhance in the postcontrast phase ${ }^{(13)}$ and may produce a chemical shift artefact at the fat-fluid interface on gradient echo sequences. ${ }^{(12)}$ Lipoma arborescens is often associated with an effusion (Figs. 10-12).

There are several differential diagnoses. One of them is synovial lipoma, which usually presents as a round or oval mass-like lesion. In the knee, synovial lipoma tends to affect the infrapatellar fat 

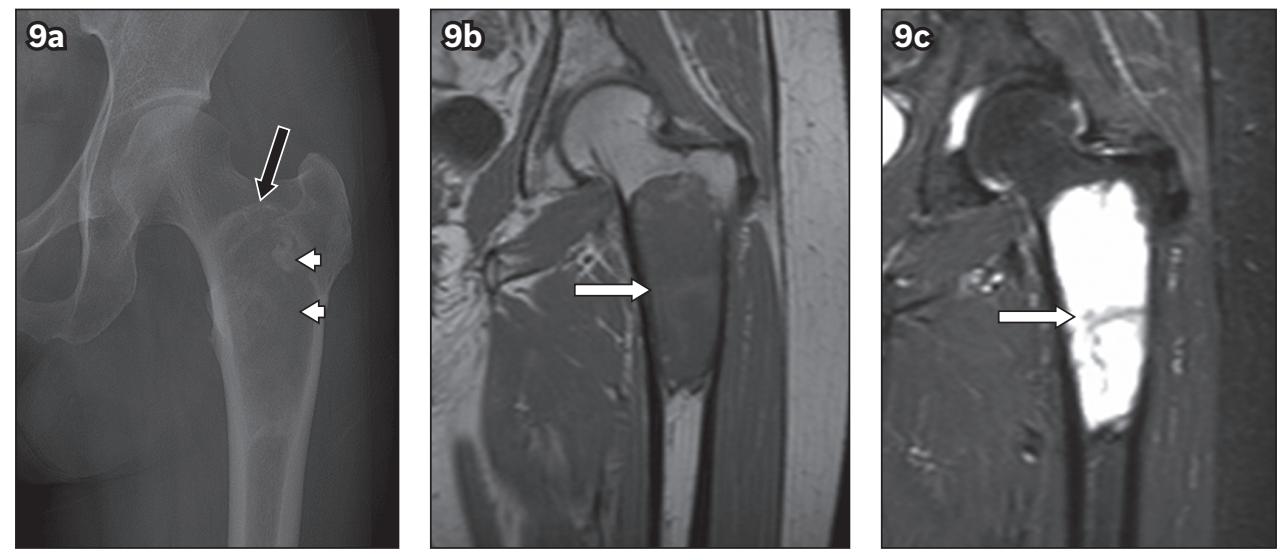

Fig. 9 Liposclerosing myxofibrous tumour at the intertrochanteric region of the femur. (a) Anteroposterior radiograph of the left hip shows a slightly expansile geographic lesion with a thin, sclerotic rim (black arrow) and a partially mineralised amorphous matrix (arrowheads). (b) T1-W MR image shows a lesion with a relatively homogeneous appearance with similar signal intensity to skeletal muscle and no hyperintense fat (white arrow). (c) Short tau inversion recovery MR image shows that the lesion is hyperintense with no fat suppression (white arrow).
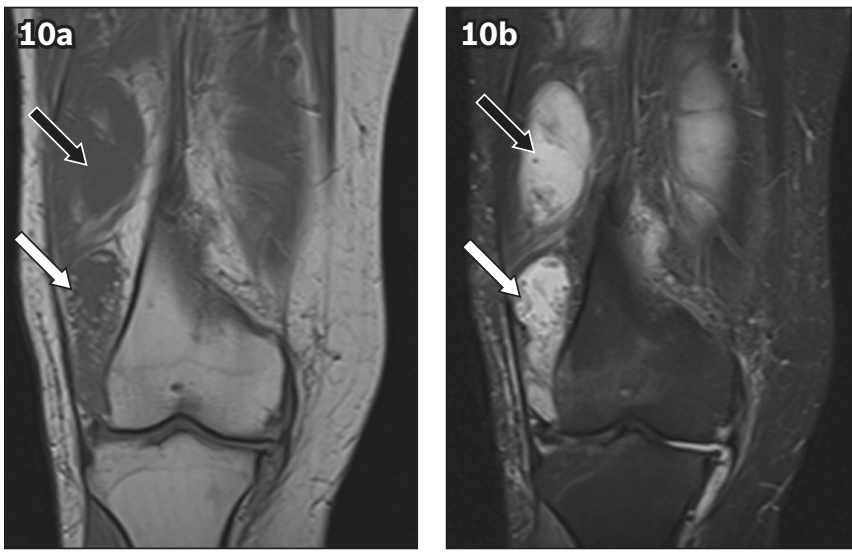

Fig. 10 Lipoma arborescens in the knee. (a) Coronal T1-W and (b) short tau inversion recovery MR images show suprapatellar effusion (black arrows) and frond-like projections of the lipomatous tissue (white arrows).
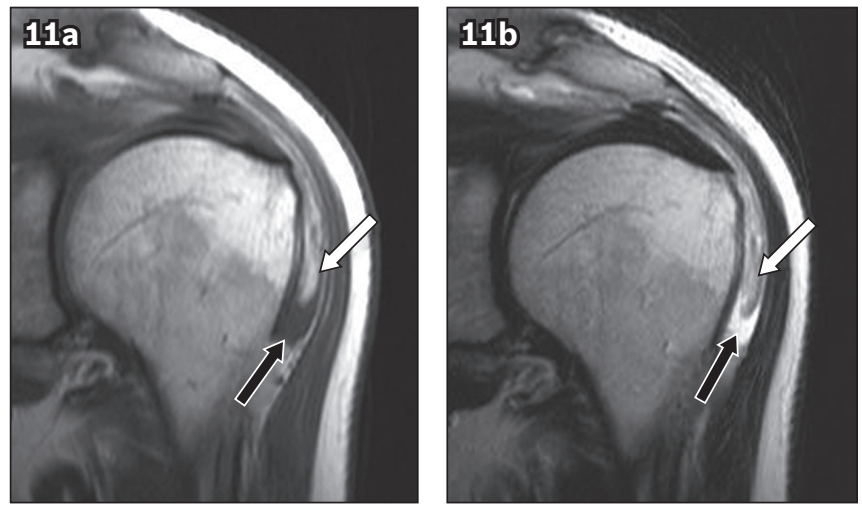

Fig. 11 Lipoma arborescens of the subacromial-subdeltoid bursa. (a) Coronal T1-W and (b) T2-W MR images show frond-like projections of the lipomatous tissue (white arrows) within the subacromial-subdeltoid bursa and an effusion (black arrows)
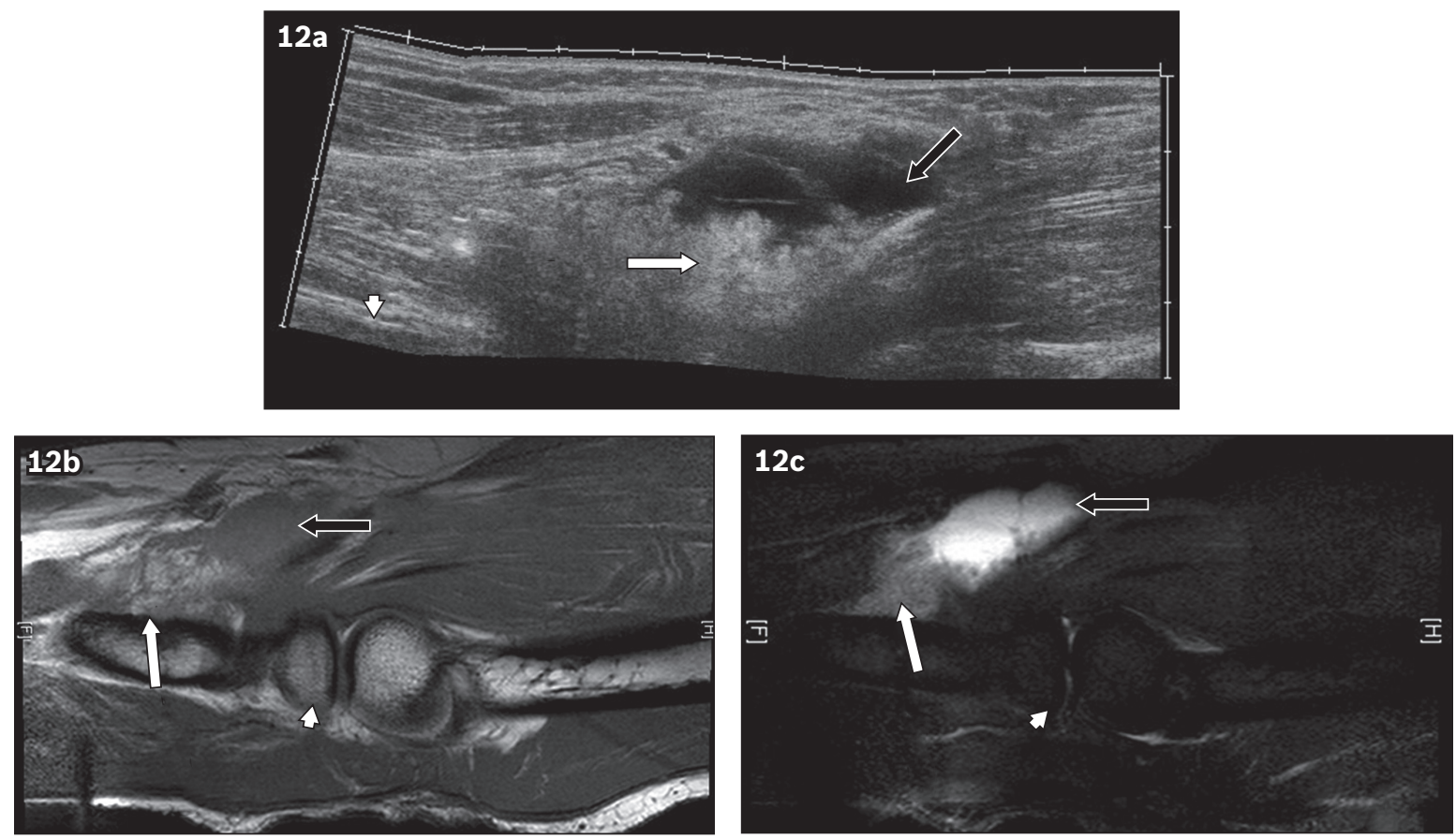

Fig. 12 Bicipitoradial lipoma arborescens. (a) Longitudinal US image of the bicipitoradial bursa shows a frond-like echogenic mass (white arrow) and an effusion (black arrow). Sagittal (b) T1-W and (c) T2-W fat-saturated MR images confirm that the frond-like mass is a lipomatous lesion (white arrows in b \& c) within the bicipitoradial bursa and an associated effusion (black arrows in b \& c). The arrowheads indicate the radius bone. 

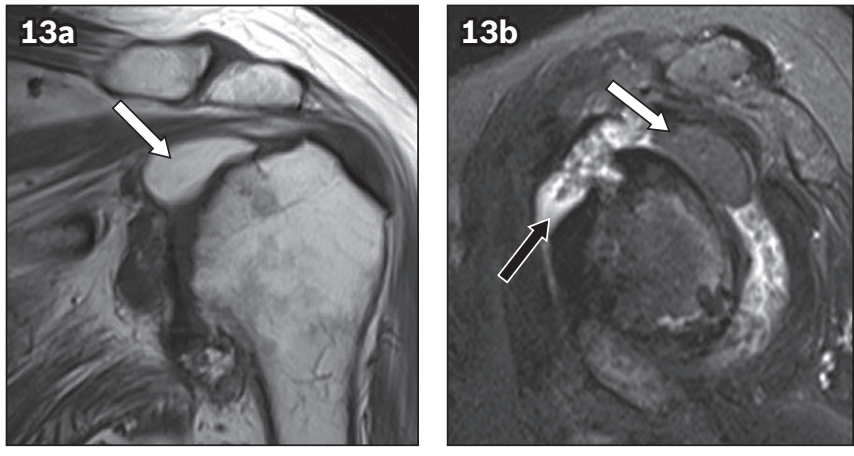

Fig. 13 Intra-articular lipoma of the glenohumeral joint. (a) Coronal T1-W MR image shows a well-defined, homogeneously hyperintense lesion (white arrow) within the glenohumeral joint, inferior to the supraspinatus tendon. (b) Sagittal T2-W fat-saturated MR image shows a homogeneously hypointense lesion (white arrow). The features are in keeping with those of a lipoma. Severe osteoarthritis with synovitis and a large joint effusion are present (black arrow).

pad rather than the suprapatellar bursa; the latter is often affected in lipoma arborescens. ${ }^{(11,12)}$ Pigmented villonodular synovitis, another differential diagnosis, contains haemosiderin, which shows a diffuse, low signal on T1- and T2-weighted images, and susceptibility on gradient echo sequences. Furthermore, it enhances in the postcontrast phase. ${ }^{(12)}$ Haemangiomas show intermediate signal on T1- and T2-weighted images, and may contain signal voids or hypointense septa. Pannus in rheumatoid arthritis has intermediate to low signal on T1- and T2-weighted images. ${ }^{(13}$

The typical treatment for lipoma arborescens is arthroscopic or open synovectomy. ${ }^{(1,12)}$

\section{INTRA-ARTICULAR LIPOMA}

Discrete intra-articular (synovial) lipoma is a very rare lesion with approximately 24 cases reported to date. ${ }^{(14)}$ Most of them occurred in the knee $(n=19)$, while one case each was reported in the following joints: hip, ${ }^{(15)}$ lumbar facet, tarsometatarsal, elbow and shoulder. ${ }^{(1,14,16,17)}$ The case of shoulder intra-articular lipoma featured in this article may be the first published case that demonstrates its MR imaging appearance in the shoulder joint (Fig. 13).

The clinical presentation of intra-articular lipoma depends on the site and size of the lesion. Patients may be asymptomatic or have complaints of dull pain due to pressure, a sudden pain related to twisting and strangulation of the lipoma, limitation of motion, crepitus and joint effusion. ${ }^{(14,16)}$

Intra-articular lipoma may not always be identified on radiographs, especially when it is small. ${ }^{(15,16)}$ If visualised, the lesion presents as a well-defined radiolucency. ${ }^{(14)} \mathrm{MR}$ imaging is the next imaging modality for investigation of this lesion. On MR imaging, the lesion is hyperintense on both T1- and T2-weighted sequences (Fig. 13). ${ }^{(16)}$ However, it can also present as a nonspecific finding on MR imaging, appearing heterogeneous with fluid-like signal within that is thought to be due to mucoid degeneration. . $^{(1,14)}$

The main differential diagnosis is lipoma arborescens, ${ }^{(14,16)}$ which is also an intra-articular lipomatous lesion. An important characteristic that differentiates the two types of lesions is the villous appearance of lipoma arborescens on all imaging modalities. In addition, on ultrasonography, lipoma arborescens may have a wave-like motion within an intra-articular effusion. ${ }^{(14)}$ Another extremely rare differential diagnosis is intra-articular angiomyolipoma, which has only one reported case in literature. This lesion was discovered in the knee, superior to the Hoffa's fat pad, and only part of the angiomyolipoma had MR imaging signal that was isointense to fat. The rest of the lesion had heterogeneous signal intensity and contained areas of fluid-like signal. ${ }^{(18)}$

Depending on its size, intra-articular lipoma can be resected arthroscopically or via open surgery. ${ }^{(14-16)}$

\section{CONCLUSION}

With the advent of advanced imaging techniques, asymptomatic, benign periarticular, bone and joint lipomatous lesions are increasingly discovered incidentally. Awareness of the imaging characteristics of these lesions at various sites of the body and on different modalities is useful. It can prevent unnecessary biopsies in the absence of aggressive features, which helps to optimise clinical management.

\section{REFERENCES}

1. Murphey MD, Carroll JF, Flemming DJ, et al. From the archives of the AFIP: benign musculoskeletal lipomatous lesions. Radiographics 2004; 24:1433-66.

2. Campbell RS, Grainger AJ, Mangham DC, et al. Intraosseous lipoma: report of 35 new cases and a review of the literature. Skeletal Radiol 2003; 32:209-22.

3. Kransdorf MJ, Murphey MD, Sweet DE. Liposclerosing myxofibrous tumor: a radiologic-pathologic-distinct fibro-osseous lesion of bone with a marked predilection for the intertrochanteric region of the femur. Radiology 1999; 212:693-8

4. Dattilo J, McCarthy EF. Liposclerosing myxofibrous tumor (LSMFT), a study of 33 patients: should it be a distinct entity? lowa Orthop J 2012; 32:35-9.

5. Howe BM, Wenger DE. Lipoma arborescens: comparison of typical and atypical disease presentations. Clin Radiol 2013; 68:1220-6.

6. Propeck T, Bullard MA, Lin J, Doi K, Martel W. Radiologic-pathologic correlation of intraosseous lipomas. AJR Am J Roentgenol 2000; 175:673-8.

7. Milgram JW. Intraosseous lipomas: radiologic and pathologic manifestations. Radiology 1988; 167:155-60.

8. Kwak HS, Lee KB, Lee SY, Han YM. On the AJR viewbox. MR findings of calcaneal intraosseous lipoma with hemorrhage. AJR Am J Roentgenol 2005; 185:1378-9.

9. Sohn $\mathrm{MH}$, Lim ST, Jeong $\mathrm{HJ}$, et al. Intraosseous lipoma in the femoral shaft mimicking a bone metastasis on bone scintigraphy. Clin Nucl Med 2009; 34:693-5.

10. Freiberg RA, Air GW, Glueck CJ, Ishikawa T, Abrams NR. Multiple intraosseous lipomas with type-IV hyperlipoproteinemia. A case report. J Bone Joint Surg Am 1974; 56:1729-32.

11. Vilanova JC, Barceló J, Villalón M, et al. MR imaging of lipoma arborescens and the associated lesions. Skeletal Radiol 2003; 32:504-9.

12. Kloen $\mathrm{P}$, Keel SB, Chandler HP, et al. Lipoma arborescens of the knee. J Bone Joint Surg Br 1998; 80:298-301.

13. Ritchie DA. MR imaging of synovial tumours and tumour-like lesions. Br J Radiol 1999; 72:212-8.

14. Poorteman L, Declercq H, Natens P, Wetzels K, Vanhoenacker F. Intra-articular synovial lipoma of the knee joint. BJR Case Rep 2015; 1:20150061.

15. Margheritini F, Villar RN, Rees D. Intra-articular lipoma of the hip. A case report. Int Orthop 1998; 22:328-9.

16. Pavithra P, Arundhathi S, Kodandaswamy CR. Intra articular synovial lipoma of the right tarsometatarsal joint: a rare case report. J Clin Diagn Res 2014; 8:FD03-4.

17. Wetson WJ. The intra-synovial fatty masses in chronic rheumatoid arthritis. $\mathrm{Br}$ J Radiol 1973; 46:213-6.

18. Bergin PF, Milchteim C, Beaulieu GP, et al. Intra-articular knee mass in a 51-year-old woman. Orthopedics 2011; 34:223 


\section{SINGAPORE MEDICAL COUNCIL CATEGORY 3B CME PROGRAMME} (Code SMJ 201709B)

Question 1. Intraosseous lipomas:

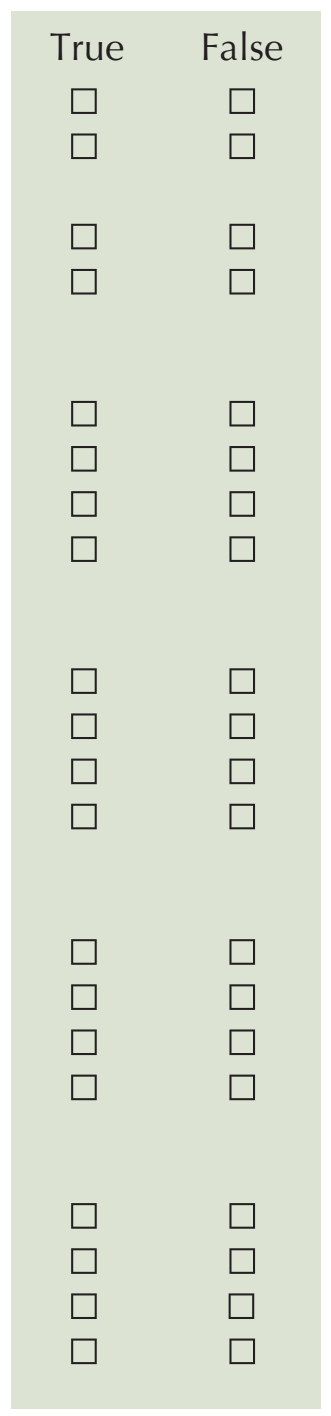

(a) Are commonly asymptomatic.

(b) Can undergo calcification and haemorrhage, and form cysts.

(c) Have lipomatous areas that, unlike fat, are hypointense on T1-weighted magnetic resonance (MR) imaging.

(d) Commonly show resorption of bone trabeculae and prominent expansion of the bone.

Question 2. Liposclerosing myxofibrous tumours:

(a) Have typical lipomatous characteristics on computed tomography and MR imaging.

(b) Commonly occur in the femur.

(c) Can undergo malignant transformation into osteosarcoma.

(d) Can appear lucent with a sclerotic margin.

Question 3. Lipoma arborescens:

(a) Are often associated with effusion and degenerative changes.

(b) Are often polyarticular.

(c) Enhance in the postcontrast phase on MR imaging.

(d) Can be differentiated from pigmented villonodular synovitis on MR imaging.

Question 4. Intra-articular lipomas:

(a) Have a villous appearance on imaging.

(b) Are hyperintense on T1- and T2-weighted sequences.

(c) Mostly occur in the knee joint.

(d) Commonly ossify.

Question 5. Benign periarticular, bone and joint lipomatous lesions:
(a) Tend to affect the elderly.
(b) May be incidentally discovered on imaging that is performed to look for another pathology.
(c) Always require a biopsy.
(d) Have an absence of radionuclide uptake.

\section{Doctor's particulars:}

Name in full: MCR no.:

Specialty: Email: 